\title{
AN OPTIMIZATION OF SOLID TRANSPORTATION PROBLEM WITH STOCHASTIC DEMAND BY LAGRANGIAN FUNCTION AND KKT CONDITIONS
}

\author{
Anjana Kuiri*, Barun Das and Sanat Kumar Mahato
}

\begin{abstract}
In this paper, a stochastic solid transportation problem (SSTP) is constructed where the demand of the item at the destinations are randomly distributed. Such SSTP is formulated with profit maximization form containing selling revenue, transportation cost and holding/shortage cost of the item. The proposed SSTP is framed as a nonlinear transportation problem which is optimized through Karush-Kuhn-Tucker (KKT) conditions of the Lagrangian function. The primary model is bifurcated into three different models for continuous and discrete demand patterns. The concavity of the objective functions is also presented here very carefully. Finally, a numerical example is illustrated to stabilize the models.
\end{abstract}

Mathematics Subject Classification. 49Q22, 90B06, 90C08.

Received May 18, 2020. Accepted November 20, 2020.

\section{INTRODUCTION}

Transportation problem (TP) is one type of Linear Programming Problem (LPP) that involves selection of most economical shipping routes and quantities for transfer of any homogeneous commodity from a number of origins/sources to a number of destinations. The solid transportation problem (STP) was first presented by Haley [10], wherein addition with sources and destinations another parameter, the mode of transportation also is considered for the transportation of heterogeneous commodities. Keeping the requirements in mind, here three kinds of constraints are taken into account, that is, source constraint in terms of availability, destination constraint in terms of demand and conveyance's capacity constraint. The STP is of much use in public distribution systems. The STP degenerates into the classical transportation problem if the number of conveyance is only one. In recent years, there have been numerous papers in this area. Most of the papers only minimize the total transportation cost. Ojha et al. [29] considered a STP for an item with fixed charge, vehicle cost and price discounted varying charge. Interested readers may consult Bit et al. [3], Jimenez and Verdegay [16] and so on. But in reality maximization of profit become a more essential objective to the decision-makers.

In many practical situations, the vagueness appeared in the parameters of TP. This is due to the lack of information about the system, insufficiency in the transportation policy, different types of unexpected factors such as lack of evidence, fluctuation in the market, artificial crisis in the market, unstable political situation,

Keywords. Solid transportation problem, stochastic demand, Lagrangian, KKT condition.

Department of Mathematics, Sidho-Kanho-Birsha University, Purulia 723104, India.

*Corresponding author: anjanakuiri@gmail.com 
etc. In such situation the stochastic nature appears to the TP and STP. The stochastic transportation problem was discussed by several researchers such as Williams [37], Holmberg [11-13], Wilson [38] and Cooper [5]. This is a variant of the ordinary (linear) transportation problem with random demand. A STP with one or more random parameters is termed as a stochastic solid transportation problem (SSTP). Yang and Feng [39] solved a bicriteria STP in stochastic environment. Quddoos et al. [30] presented a multi-choice stochastic transportation problem involving a general form of distribution. Halder et al. [9] solved a solid transportation problem through fuzzy ranking. Ojha et al. [28] designed a multi-objective SSTP for breakable items using analytic hierarchy process. Habiba and Quddoos [8] discussed a multi-objective stochastic transportation problem with interval cost coefficients. Yang et al. [40] introduced the reduction methods of type-2 uncertain variables to STP. Jana et al. [14] described the profit maximization STP with Gaussian type-2 fuzzy environments. Anukokila et al. [2] presented a goal programming approach for solving multi-objective fractional transportation problem with fuzzy parameters. Ojha et al. [27] framed multi-objective STP as a geometric programming problem. Das et al. [7] proposed a new approach for solving fully fuzzy linear fractional programming problems using the multi-objective linear programming method/technique. Jahanshahloo et al. [15] presented a solution procedure for multi-objective linear fractional programming problem based on goal programming and data envelopment analysis. Bhurjee and Panda [4], Roy and Midya [32], Roy et al. [35] developed a multi-objective transportation problem with variety of conceptions. Many researchers $[6,18,19]$ described STP in different environments. In 2010, Nagarjan and Jeyaraman [26] solved a solid transportation problem by using fuzzy approach under stochastic environment. Due to the random nature of the demand, two situations can occur-either demand is less than the total commodity received or demand is more than that. Such respective situations lead to holding and shortage of the commodity at the destinations.

Because of flexibility and uni-modality, the logistic distribution has a wide application in any real life decision making problem. It can be used to model the data exhibiting having some skewness property. The probability density function of a logistic distribution with location and scale parameters $\alpha$ and $\beta$ for a random variable $t$ is described by

$$
\phi(t)=\frac{1}{\beta} \frac{e^{-\left(\frac{t-\alpha}{\beta}\right)}}{\left[1+e^{-\left(\frac{t-\alpha}{\beta}\right)}\right]^{2}}, 0 \leq t<\infty, 0 \leq \alpha<\infty, \text { and } \beta>0 .
$$

The logistic distribution is more popular for the economical modeling of those items whose demand function is concave in shape and the item is of high-risk management. The similarity of Poisson distribution finds in the exponential distribution. It occurs in the situation of time until the failure of a part and separation between random events happen. The probability density function of an exponential distribution is described as

$$
\phi(t)=\lambda e^{-\lambda t}, t>0, \lambda>0 .
$$

These types of functions present by reverse-J shaped. It is often termed as compound Poisson distribution. In real-life situations, it is observed that the demand of an item is not known precisely. To stimulate demand, we have considered uncertain demand with stochastic nature. Such type of demand is found for those items whose demand slowly decreasing. The TP with stochastic demand is a special version of the stochastic linear programming problem. It has many economic applications. The stochastic transportation problem has been discussed in many papers $[21,23,25,34]$ in several ways and solved by different methods. Mahapatra et al. $[20,22]$ and Roy et al. $[31,33]$ presented a procedure to solve the multi-choice transportation problem where they have converted the multi-choice transportation problem into a standard mathematical programming through the selection of binary variables, bounds for binary codes, and restriction of binary codes using auxiliary constraints. The optimality of the constrained optimization problem has been found out in descent directions. The KKT conditions based on Lagrangian function are the gradient search direction condition which are necessary for the optimization of the constrained problem. These conditions also may transform into sufficient conditions if the objective function satisfies the convexity/concavity condition. Kim et al. [17] make a note on second-order 
TABLE 1. Comparisons of the problem with existing transportation problem (TP).

\begin{tabular}{|c|c|c|c|c|}
\hline References & Type of TP & $\begin{array}{l}\text { Demand of } \\
\text { the item }\end{array}$ & Formulation type & Solution procedure \\
\hline Williams [37] & $2 \mathrm{D}$ & Stochastic & Cost minimization & Lingo Software \\
\hline Das et al. [6] & $3 \mathrm{D}$ & Constant & do & Fuzzy programming \\
\hline Bit et al. $[3]$ & $3 \mathrm{D}$ & do & do & do \\
\hline Nagarjan and & $3 \mathrm{D}$ & do & do & Chance programming \\
\hline \multicolumn{5}{|l|}{ Jeyaraman [26] } \\
\hline Ojha et al. [29] & $3 \mathrm{D}$ & do & do & Genetic algorithm \\
\hline Mahapatra [22] & $2 \mathrm{D}$ & Stochastic & do & Lingo Software \\
\hline Mahapatra [20] & $2 \mathrm{D}$ & do & do & do \\
\hline $\begin{array}{l}\text { Holemberg and Tuy } \\
{[13]}\end{array}$ & $2 \mathrm{D}$ & do & do & Branch and bound procedure \\
\hline Jana et al. $[14]$ & $3 \mathrm{D}$ & Constant & Profit maximization & Genetic algorithm \\
\hline Wilson [38] & $2 \mathrm{D}$ & Stochastic & Cost minimization & Primal-dual algorithm \\
\hline This paper & $3 \mathrm{D}$ & Stochastic & $\begin{array}{l}\text { Profit maximization } \\
\text { in Lagrangian form }\end{array}$ & $\begin{array}{l}\text { KKT conditions then by Lingo } \\
\text { Software }\end{array}$ \\
\hline
\end{tabular}

Karush-Kuhn-Tucker necessary optimality conditions for smooth vector optimization problems. Recently Maity et al. [24] and Samanta et al. [36] presented the transportation problem in a spectacular direction.

The major contributions of the paper are as follows.

- Here, a STP is considered with random demand. Due to the randomness of the demand, there may arise two mutually exclusive events (situations). Demand is either less than the total receiving amount (this leads to holding situation) or greater than that (leads to shortage situation).

- The STP is constructed here on the objective of profit maximization in the decision maker's point of view, containing the terms of selling revenue, transportation cost, expected holding cost or expected shortage cost.

- The randomness of the demand also felt an effect on the STP constraints.

- To fulfil the random criteria of the demand, here logistic and exponential distributions are taken for a continuous case and a discrete probability distribution is also taken into consideration. The logistic demand met the scenario when demand varies with time of use, season and socio-economic pattern of the consumers. And the exponential distribution pattern seems to commodities whose demand starts from the non-negative quantity and tends to zero at the end of the period.

- Here, STP is optimized by the formulation of Lagrangian function with Lagrange multiplier and then by Kuhn Tuckher's optimality conditions.

The research gap of this article with the existing literatures on the TP is shown in the following Table 1.

\section{Non-Linear optimization method (KKT approach)}

Consider the constrained maximization problem as

$$
\begin{aligned}
\operatorname{Max} & f\left(x_{1}, x_{2}, \ldots, x_{n}\right) \\
\text { subject to } & g_{i}\left(x_{1}, x_{2}, \ldots, x_{n}\right) \geq 0 ; \quad i=1,2, \ldots, m \\
& h_{j}\left(x_{1}, x_{2}, \ldots, x_{n}\right)=0 ; \quad j=1,2, \ldots, n .
\end{aligned}
$$

Then the Lagrangian function of the above problem is

$$
L(x, \mu, \lambda)=f(x)+\sum_{i=1}^{m} \mu_{i} g_{i}(x)+\sum_{j=1}^{n} \lambda_{j} h_{j}(x) .
$$


Assume that $f, g_{i}, h_{j}$ are differentiable. If the function $f\left(x^{*}\right)$ attains local maximum at point $x^{*}$, then the Lagrange multipliers $\mu_{i}, \lambda_{j}$ satisfied the following conditions:

(i) $\frac{\partial L}{\partial x^{*}}=\frac{\partial f}{\partial x^{*}}+\sum_{i=1}^{m} \mu_{i} \frac{\partial g_{i}}{\partial x^{*}}+\sum_{j=1}^{n} \lambda_{j} \frac{\partial h_{j}}{\partial x^{*}}=0$ (stationarity condition).

(ii) $\mu_{i} g_{i}\left(x_{1}^{*}, x_{2}^{*}, \ldots, x_{n}^{*}\right)=0, i=1,2, \ldots, m$ (complementary slackness condition).

(iii) $\lambda_{j} h_{j}\left(x_{1}^{*}, x_{2}^{*}, \ldots, x_{n}^{*}\right)=0, j=1,2, \ldots, n$ (complementary slackness condition).

(iv) $g_{i}\left(x_{1}^{*}, x_{2}^{*}, \ldots, x_{n}^{*}\right) \geq 0, i=1,2, \ldots, m$ (primal feasibility condition).

(v) $h_{j}\left(x_{1}^{*}, x_{2}^{*}, \ldots, x_{n}^{*}\right) \geq 0, j=1,2, \ldots, n$ (primal feasibility condition).

(vi) $\mu_{i}, \lambda_{j} \geq 0$ (dual feasibility condition).

In other words, the conditions (i)-(vi) are necessary conditions for a local maximum of the problem. Also, conditions (i)-(vi) are called the Karush-Kuhn-Tucker (KKT) conditions.

In the particular case of $m=0$, i.e., when there are no inequality constraints, the KKT conditions turn into the Lagrangian conditions, and the KKT multipliers are called Lagrange multipliers.

Karush-Kuhn-Tucker conditions are first-order necessary conditions for an optimal solution of nonlinear programming, provided that some regularity conditions are satisfied.

\section{MOdEL DESCRIPTION AND FORMULATION}

\subsection{Assumption}

In this SSTP the following assumptions and notations are made:

(a) Availability $\left(a_{i}\right)$ of the $i$ th origin is finite and known.

(b) The demand $d_{j}$ of the item at the $j$ th destination is random in nature and followed probabilities density function $\phi_{j}\left(d_{j}\right)$.

(c) The actual received quantity $y_{j}$ by the $j$ th destination may be lower or higher than the expected demand $E\left(d_{j}\right)$. Depending on that situation their may be shortage or holding of the item at the destination $j$. In this regard, shortage and holding cost are taken here.

(d) Capacity $\left(e_{k}\right)$ of the $k$ th conveyance is finite and known.

(e) The cost $\left(c_{i j k}\right)$ for transporting one unit item from sources $i$ to destination $j$ by conveyance $k$ is finite and known.

(f) The amount of transportation $\left(x_{i j k}\right)$ from sources $i$ to destination $j$ with the aid of conveyance $k$ is finite and decision variable.

(g) The unit holding cost and shortage cost of the item at the $j$ th destination are $h_{j}$ and $p_{j}$ respectively, which are finite and known.

(h) The unit selling price of the item at the $j$ th destination $\left(S_{j}\right)$ is finite and known.

\subsection{Formulation of the model}

We consider a STP with $m$ supply node, $n$ demand node and $k$ capacity conveyances. If $x_{i j k}$ is the shifted amount from $i$ th supply to $j$ th destination through the $k$ th conveyance and $y_{j}$ be the total amount that is shipped to the demand point $j$ from all supply node then, $y_{j}=\sum_{i=1}^{m} \sum_{k=1}^{l} x_{i j k}$. Then either $y_{j} \geq E\left(d_{j}\right)$ or $y_{j}<E\left(d_{j}\right)$.

There may be two cases:

Case I. Total transported amount is greater than or equal to the random demand.

In this case, there will be a surplus and hence holding cost arises. So the expected holding charge is paid at the destination $j$, which is equal to $h_{j} \int_{0}^{y_{j}}\left(y_{j}-t\right) \phi_{j}(t) \mathrm{d} t$ or $h_{j} \sum_{t<y_{j}}\left(y_{j}-t\right) P\left(d_{j}=t\right), h_{j}>0$, where $\phi_{j}(t)$ is the probability density function (p.d.f) of the random variable $d_{j}$. 
Case II. Total transported amount is less than the random demand.

In this case, there will be a shortage, so the destination manager need to pay the expected shortage cost $p_{j} \int_{y_{j}}^{\infty}\left(t-y_{j}\right) \phi_{j}(t) \mathrm{d} t$ or $p_{j} \sum_{t>y_{j}}\left(t-y_{j}\right) P\left(d_{j}=t\right), p_{j}>0$.

Since the above two events are mutually exclusive i.e., cannot occur simultaneously, so probabilitically, the expected cost consists the sum of the individual.

For the whole system, the total holding/shortage cost

$$
\sum_{j=1}^{n}\left[h_{j} \int_{0}^{y_{j}}\left(y_{j}-t\right) \phi_{j}(t) \mathrm{d} t+p_{j} \int_{y_{j}}^{\infty}\left(t-y_{j}\right) \phi_{j}(t) \mathrm{d} t\right]
$$

or

$$
\sum_{j=1}^{n}\left[h_{j} \sum_{t<y_{j}}\left(y_{j}-t\right) P\left(d_{j}=t\right)+p_{j} \sum_{t>y_{j}}\left(t-y_{j}\right) P\left(d_{j}=t\right)\right]
$$

is a convex function $(c f .[13])$.

So the expected profit function $(Z)$ of the decision maker is given by:

$$
\begin{aligned}
\langle\text { Expected Profit }\rangle= & \langle\text { Selling revenue }\rangle-\langle\text { Transportation cost }\rangle \\
& -\langle\text { Expected holding cost }\rangle-\langle\text { Expected shortage cost }\rangle .
\end{aligned}
$$

The aim of this problem is to determine the optimum expected profit of the transportation system for delivering homogenous/heterogeneous commodities from various sources to different destinations in different conveyances. Mathematically,

$$
\begin{aligned}
\operatorname{Max} Z= & \sum_{j=1}^{n} S_{j} y_{j}-\sum_{j=1}^{n} \sum_{i=1}^{m} \sum_{k=1}^{l} c_{i j k} x_{i j k}-\sum_{j=1}^{n}\left[h_{j} \int_{0}^{y_{j}}\left(y_{j}-t\right) \phi_{j}(t) \mathrm{d} t\right] \\
& -\sum_{j=1}^{n}\left[p_{j} \int_{y_{j}}^{\infty}\left(t-y_{j}\right) \phi_{j}(t) \mathrm{d} t\right] \quad \text { (for continuous case) }
\end{aligned}
$$

and

$$
\begin{aligned}
\operatorname{Max} Z= & \sum_{j=1}^{n} S_{j} y_{j}-\sum_{j=1}^{n} \sum_{i=1}^{m} \sum_{k=1}^{l} c_{i j k} x_{i j k}-\sum_{j=1}^{n}\left[h_{j} \sum_{t<y_{j}}\left(y_{j}-t\right) P\left(d_{j}=t\right)\right] \\
& -\sum_{j=1}^{n}\left[p_{j} \sum_{t>y_{j}}\left(t-y_{j}\right) P\left(d_{j}=t\right)\right] \quad \text { (for discrete case). }
\end{aligned}
$$

After simplification and neglecting the constant term [1], equations (3.1) and (3.2) are equivalent to

$$
\begin{aligned}
& \operatorname{Max} Z=\sum_{j=1}^{n} S_{j} y_{j}-\sum_{j=1}^{n} \sum_{i=1}^{m} \sum_{k=1}^{l} c_{i j k} x_{i j k}-\sum_{j=1}^{n}\left(h_{j}+p_{j}\right) \int_{0}^{y_{j}}\left(y_{j}-t\right) \phi_{j}(t) \mathrm{d} t+\sum_{j=1}^{n} p_{j} y_{j} \\
& \operatorname{Max} Z=\sum_{j=1}^{n} S_{j} y_{j}-\sum_{j=1}^{n} \sum_{i=1}^{m} \sum_{k=1}^{l} c_{i j k} x_{i j k}-\sum_{j=1}^{n}\left(h_{j}+p_{j}\right) \sum_{r<y_{j}}\left(y_{j}-r\right) \mu_{j}^{r}+\sum_{j=1}^{n} p_{j} y_{j} .
\end{aligned}
$$

And the constraints of the proposed stochastic solid transportation problem (SSTP) are

$$
\text { Capacity constraints of the origins: } \sum_{j=1}^{n} \sum_{k=1}^{l} x_{i j k} \leq a_{i} \text { for } i=1,2, \ldots, m
$$


Demand constraints at the destination: $\sum_{i=1}^{m} \sum_{k=1}^{l} x_{i j k}=y_{j}$ for $j=1,2, \ldots, n$

Capacity constraints of the conveyances: $\sum_{i=1}^{m} \sum_{j=1}^{n} x_{i j k} \leq e_{k}$ for $k=1,2, \ldots, l$

And feasibility constraints amount of transportation: $x_{i j k} \geq 0 \forall i, j, k$.

Any set of allocations which satisfied the equations (3.4)-(3.7) is called a feasible solution of the solid transportation problem (without boundedness of the demand). A feasible solution to that problem is said to be basic, i.e., BFS if the set of allocations are independent. The BFS which maximizes equations (3.3a) and (3.3b) is called an optimal basic feasible solution. Again, if the number of non zero variables is less than $m+n+l-2$, then the problem is called degenerate.

\section{Model With Distribution FUnCtions}

Here, the model is bifurcated into different sub-models, based on different types (continuous and discrete) of a probability distribution of the random demand.

- Sub-Model 1. Let the random demands $b_{j}(j=1,2, \ldots, n)$ of the $j$ th destinations followed logistic distribution with the following probability density function $\phi\left(b_{j}\right)$. The logistic distribution is the "normal"-shaped pattern of its cumulative distribution function (the logistic function) and quantile function (the logit function) have been extensively used in different areas.

$$
\phi\left(b_{j}\right)=\frac{1}{\beta_{j}} \frac{e^{-\left(\frac{b_{j}-\alpha_{j}}{\beta_{j}}\right)}}{\left[1+e^{-\left(\frac{b_{j}-\alpha_{j}}{\beta_{j}}\right)}\right]^{2}}, 0 \leq b_{j}<\infty, 0 \leq \alpha_{j}<\infty, \text { and } \beta_{j}>0 .
$$

Then equation (3.3a) becomes

$$
\begin{aligned}
\operatorname{Max} Z= & \sum_{j=1}^{n} S_{j} y_{j}-\sum_{j=1}^{n} \sum_{i=1}^{m} \sum_{k=1}^{l} c_{i j k} x_{i j k} \\
& -\sum_{j=1}^{n}\left(h_{j}+p_{j}\right)\left\{y_{j} \frac{e^{\frac{\alpha_{j}}{\beta_{j}}}}{1+e^{\frac{\alpha_{j}}{\beta_{j}}}}+\beta_{j} \log \frac{1+e^{\frac{\left(\alpha_{j}-y_{j}\right)}{\beta_{j}}}}{1+e^{\frac{\alpha_{j}}{\beta_{j}}}}\right\}+\sum_{j=1}^{n} p_{j} y_{j}
\end{aligned}
$$

subject to the constraints (3.4)-(3.7).

The Lagrange's function of the above problem is

$$
\begin{aligned}
L(X, u, v, z)= & \sum_{j=1}^{n} S_{j} y_{j}-\sum_{j=1}^{n} \sum_{i=1}^{m} \sum_{k=1}^{l} c_{i j k} x_{i j k} \\
& -\sum_{j=1}^{n}\left(h_{j}+p_{j}\right)\left\{y_{j} \frac{e^{\frac{\alpha_{j}}{\beta_{j}}}}{1+e^{\frac{\alpha_{j}}{\beta_{j}}}}+\beta_{j} \log \frac{1+e^{\frac{\left(\alpha_{j}-y_{j}\right)}{\beta_{j}}}}{1+e^{\frac{\alpha_{j}}{\beta_{j}}}}\right\}+\sum_{j=1}^{n} p_{j} y_{j} \\
& +\sum_{i=1}^{m} u_{i} \sum_{j=1}^{n} \sum_{k=1}^{l}\left(a_{i}-x_{i j k}\right)+\sum_{k=1}^{l} v_{k} \sum_{i=1}^{m} \sum_{j=1}^{n}\left(e_{k}-x_{i j k}\right) \\
& +\sum_{i=1}^{m} \sum_{j=1}^{n} \sum_{k=1}^{l} z_{i j k} x_{i j k}
\end{aligned}
$$


where the variable $u_{i}, v_{k}, z_{i j k}$ are Lagrange multipliers and $u_{i}, v_{k}, z_{i j k} \geq 0$. The KKT conditions for the problem (4.2) are

$$
\left\{\begin{array}{l}
\frac{\partial L}{\partial x_{i j k}}=c_{i j k}-u_{i}-v_{k}+z_{i j k}=0 \\
\sum_{i=1}^{m} u_{i}\left(\sum_{j=1}^{n} \sum_{k=1}^{l}\left(a_{i}-x_{i j k}\right)\right)=0 \\
\sum_{k=1}^{l} v_{k}\left(\sum_{i=1}^{m} \sum_{j=1}^{n}\left(e_{k}-x_{i j k}\right)\right)=0 \\
\sum_{i=1}^{m} \sum_{j=1}^{n} \sum_{k=1}^{l} z_{i j k} x_{i j k}=0
\end{array}\right.
$$

Now, the KKT conditions, lead the optimization problem to

$$
\left\{\begin{array}{l}
\operatorname{Max} Z=\sum_{j=1}^{n} S_{j} y_{j}-\sum_{j=1}^{n} \sum_{i=1}^{m} \sum_{k=1}^{l} c_{i j k} x_{i j k}-\sum_{j=1}^{n}\left(h_{j}+p_{j}\right)\left\{y_{j} \frac{e^{\frac{\alpha_{j}}{\beta_{j}}}}{1+e^{\frac{\alpha_{j}}{\beta_{j}}}}+\beta_{j} \log \frac{1+e^{\frac{\left(\alpha_{j}-y_{j}\right)}{\beta_{j}}}}{1+e^{\frac{\alpha_{j}}{\beta_{j}}}}\right\}+\sum_{j=1}^{n} p_{j} y_{j} \\
\text { subject to } \\
c_{i j k}-u_{i}-v_{k} \leq 0 \\
x_{i j k}\left(c_{i j k}-u_{i}-v_{k}\right)=0 \\
\sum_{j=1}^{n} \sum_{k=1}^{l} x_{i j k} \leq a_{i} \text { for } i=1,2, \ldots, m \\
\sum_{i=1}^{m} \sum_{j=1}^{n} x_{i j k} \leq e_{k} \text { for } k=1,2, \ldots, l \\
x_{i j k} \geq 0 \forall i, j, k
\end{array} .\right.
$$

Proposition 4.1. The objective function $Z$ is concave with respect to $y_{j}$ and $x_{i j k}$.

Proof. To show the objective function $Z$ is concave, we have differentiate the function $Z$ (presence in Eq. (4.1)), partially with respect to $y_{j}$ and $x_{i j k}$ respectively. Then,

$$
\begin{aligned}
\frac{\partial Z}{\partial y_{j}} & =S_{j}-\left(h_{j}+p_{j}\right)\left\{\frac{e^{\frac{\alpha_{j}}{\beta_{j}}}}{1+e^{\frac{\alpha_{j}}{\beta_{j}}}}-\frac{e^{\frac{\left(\alpha_{j}-y_{j}\right)}{\beta_{j}}}}{1+e^{\frac{\left(\alpha_{j}-y_{j}\right)}{\beta_{j}}}}\right\}-p_{j} \\
\frac{\partial^{2} Z}{\partial y_{j}^{2}} & =-\left(h_{j}+p_{j}\right) \frac{e^{\frac{\left(\alpha_{j}-y_{j}\right)}{\beta_{j}}}}{\beta_{j}\left[1+e^{\frac{\left(\alpha_{j}-y_{j}\right)}{\beta_{j}}}\right]^{2}} .
\end{aligned}
$$


Similarly,

$$
\begin{aligned}
\frac{\partial^{2} Z}{\partial x_{i j k}^{2}}= & -\left(h_{j}+p_{j}\right)\left\{\left(\frac{e^{\frac{\alpha_{j}}{\beta_{j}}}}{1+e^{\frac{\alpha_{j}}{\beta_{j}}}}-\frac{e^{\frac{\left(\alpha_{j}-y_{j}\right)}{\beta_{j}}}}{1+e^{\frac{\left(\alpha_{j}-y_{j}\right)}{\beta_{j}}}}\right) \frac{\partial^{2} y_{j}}{\partial x_{i j k}^{2}}\right. \\
& \left.-\frac{e^{\frac{\alpha_{j}-y_{j}}{\beta_{j}}}}{\beta_{j}\left[1+e^{\frac{\left(\alpha_{j}-y_{j}\right)}{\beta_{j}}}\right]^{2}}\left(\frac{\partial y_{j}}{\partial x_{i j k}}\right)^{2}\right\}-\left(p_{j}-S_{j}\right) \frac{\partial^{2} y_{j}}{\partial x_{i j k}^{2}} .
\end{aligned}
$$

As $h_{j}, p_{j}, \beta_{j}$ are positive parameters. From the expression, it is clear that $\frac{\partial^{2} Z}{\partial y_{j}^{2}}<0$. Hence we conclude that $Z$ is concave with respect to $y_{j}$. By similar arguments $\frac{\partial^{2} Z}{\partial x_{i j k}^{2}}<0$. So $Z$ is concave function with respect to $x_{i j k}$ also. Hence the objective function $Z$ is concave with respect to $y_{j}$ and $x_{i j k}$.

- Sub-Model 2. When demands $b_{j}(j=1,2, \ldots, n)$ follow exponential distribution with probability density function

$$
\phi\left(b_{j}\right)=\lambda_{j} e^{-\lambda_{j} b_{j}}, b_{j}>0, \lambda_{j}>0 .
$$

The exponential distribution is one of the widely used distribution function as a demand function in the decision making problems.

Then the objective function (3.3a) becomes

$$
\operatorname{Max} Z=\sum_{j=1}^{n} S_{j} y_{j}-\sum_{j=1}^{n} \sum_{i=1}^{m} \sum_{k=1}^{l} c_{i j k} x_{i j k}-\sum_{j=1}^{n}\left(h_{j}+p_{j}\right)\left\{y_{j}+\frac{e^{-\lambda_{j} y_{j}}-1}{\lambda_{j}}\right\}+\sum_{j=1}^{n} p_{j} y_{j}
$$

subject to the constraints (3.4)-(3.7).

The Lagrange's function of the above problem is

$$
\begin{aligned}
L(X, u, v, z)= & \sum_{j=1}^{n} S_{j} y_{j}-\sum_{j=1}^{n} \sum_{i=1}^{m} \sum_{k=1}^{l} c_{i j k} x_{i j k}-\sum_{j=1}^{n}\left(h_{j}+p_{j}\right)\left\{y_{j}+\frac{e^{-\lambda_{j} y_{j}}-1}{\lambda_{j}}\right\}+\sum_{j=1}^{n} p_{j} y_{j} \\
& +\sum_{i=1}^{m} u_{i} \sum_{j=1}^{n} \sum_{k=1}^{l}\left(a_{i}-x_{i j k}\right)+\sum_{k=1}^{l} v_{k} \sum_{i=1}^{m} \sum_{j=1}^{n}\left(e_{k}-x_{i j k}\right) \\
& +\sum_{i=1}^{m} \sum_{j=1}^{n} \sum_{k=1}^{l} z_{i j k} x_{i j k}
\end{aligned}
$$

where the variable $u_{i}, v_{k}, z_{i j k}$ are Lagrange multipliers and $u_{i}, v_{k}, z_{i j k} \geq 0$. The KKT conditions for the problem (4.4) are

$$
\left\{\begin{array}{l}
\frac{\partial L}{\partial x_{i j k}}=c_{i j k}-u_{i}-v_{k}+z_{i j k}=0 \\
\sum_{i=1}^{m} u_{i}\left(\sum_{j=1}^{n} \sum_{k=1}^{l}\left(a_{i}-x_{i j k}\right)\right)=0 \\
\sum_{k=1}^{l} v_{k}\left(\sum_{i=1}^{m} \sum_{j=1}^{n}\left(e_{k}-x_{i j k}\right)\right)=0 \\
\sum_{i=1}^{m} \sum_{j=1}^{n} \sum_{k=1}^{l} z_{i j k} x_{i j k}=0
\end{array} .\right.
$$


Now, the KKT conditions, lead the optimization problem to

$$
\left\{\begin{array}{l}
\operatorname{Max} Z=\sum_{j=1}^{n} S_{j} y_{j}-\sum_{j=1}^{n} \sum_{i=1}^{m} \sum_{k=1}^{l} c_{i j k} x_{i j k}-\sum_{j=1}^{n}\left(h_{j}+p_{j}\right)\left\{y_{j}+\frac{e^{-\lambda_{j} y_{j}}-1}{\lambda_{j}}\right\}+\sum_{j=1}^{n} p_{j} y_{j} \\
\text { subject to } \\
c_{i j k}-u_{i}-v_{k} \leq 0 \\
x_{i j k}\left(c_{i j k}-u_{i}-v_{k}\right)=0 \\
\sum_{j=1}^{n} \sum_{k=1}^{l} x_{i j k} \leq a_{i} \text { for } i=1,2, \ldots, m \\
\sum_{i=1}^{m} \sum_{j=1}^{n} x_{i j k} \leq e_{k} \text { for } k=1,2, \ldots, l \\
x_{i j k} \geq 0 \forall i, j, k
\end{array} .\right.
$$

Proposition 4.2. The objective function $Z$ is concave with respect to $y_{j}$ and $x_{i j k}$.

Proof. To show the objective function $Z$ is concave with respect to $y_{j}$ and $x_{i j k}$, we have differentiate $Z$ partially twice with respect to $y_{j}$ and $x_{i j k}$ respectively, as

$$
\begin{aligned}
\frac{\partial Z}{\partial y_{j}} & =S_{j}-\left(h_{j}+p_{j}\right)\left\{1-e^{-\lambda_{j} y_{j}}\right\}-p_{j} \\
\frac{\partial^{2} Z}{\partial y_{j}^{2}} & =-\left(h_{j}+p_{j}\right) \lambda_{j} e^{-\lambda_{j} y_{j}} .
\end{aligned}
$$

Similarly,

$$
\frac{\partial^{2} Z}{\partial x_{i j k}^{2}}=-\left(h_{j}+p_{j}\right)\left\{\left(1-e^{-\lambda_{j} y_{j}}\right) \frac{\partial^{2} y_{j}}{\partial x_{i j k}^{2}}-\lambda_{j} e^{-\lambda_{j} y_{j}}\left(\frac{\partial y_{j}}{\partial x_{i j k}}\right)^{2}\right\}-\left(p_{j}-S_{j}\right) \frac{\partial^{2} y_{j}}{\partial x_{i j k}^{2}}
$$

As $h_{j}, p_{j}$ are positive parameters. Obviously $\frac{\partial^{2} Z}{\partial y_{j}^{2}}<0$. Hence we conclude that $Z$ is concave with respect to $y_{j}$. By similar arguments, $\frac{\partial^{2} Z}{\partial x_{i j k}^{2}}<0$, so $Z$ is concave function with respect to $x_{i j k}$ also. Hence the objective function $Z$ is concave in nature with respect to $y_{j}$ and $x_{i j k}$.

- Sub-Model 3. Let the demands $b_{j}$ of the $j$ th destination has a discrete distribution, i.e., demand of the item are frequently accept some real values $(r)$ with certain probabilities $\left(\mu_{j}^{r}\right)$, i.e., $P\left(d_{j}=r\right)=\mu_{j}^{r}, r=1,2, \ldots, K$. Where $1,2, \ldots, K$ denotes the event space of the demand $d_{j}$ at $j$ th destination and $\sum_{r=1}^{K} \mu_{j}^{r}=1$.

Then the equation $(3.3 \mathrm{~b})$ becomes

$$
\operatorname{Max} Z=\sum_{j=1}^{n} S_{j} y_{j}-\sum_{j=1}^{n} \sum_{i=1}^{m} \sum_{k=1}^{l} c_{i j k} x_{i j k}-\sum_{j=1}^{n}\left(h_{j}+p_{j}\right) \sum_{r<y_{j}}\left(y_{j}-r\right) \mu_{j}^{r}+\sum_{j=1}^{n} p_{j} y_{j}
$$

subject to the constraints (3.4)-(3.7) 
Now, the Lagrangian function of (4.5) is

$$
\begin{aligned}
L(X, u, v, z)= & \sum_{j=1}^{n} S_{j} y_{j}-\sum_{j=1}^{n} \sum_{i=1}^{m} \sum_{k=1}^{l} c_{i j k} x_{i j k}-\sum_{j=1}^{n}\left(h_{j}+p_{j}\right) \sum_{r<y_{j}}\left(y_{j}-r\right) \mu_{j}^{r}+\sum_{j=1}^{n} p_{j} y_{j} \\
& +\sum_{i=1}^{m} u_{i} \sum_{j=1}^{n} \sum_{k=1}^{l}\left(a_{i}-x_{i j k}\right)+\sum_{k=1}^{l} v_{k} \sum_{i=1}^{m} \sum_{j=1}^{n}\left(e_{k}-x_{i j k}\right) \\
& +\sum_{i=1}^{m} \sum_{j=1}^{n} \sum_{k=1}^{l} z_{i j k} x_{i j k}
\end{aligned}
$$

where the variable $u_{i}, v_{k}, z_{i j k}$ are Lagrange multipliers and $u_{i}, v_{k}, z_{i j k} \geq 0$. The KKT conditions for the problem (4.5) are

$$
\left\{\begin{array}{l}
\frac{\partial L}{\partial x_{i j k}}=c_{i j k}-u_{i}-v_{k}+z_{i j k}=0 \\
\sum_{i=1}^{m} u_{i}\left(\sum_{j=1}^{n} \sum_{k=1}^{l}\left(a_{i}-x_{i j k}\right)\right)=0 \\
\sum_{k=1}^{l} v_{k}\left(\sum_{i=1}^{m} \sum_{j=1}^{n}\left(e_{k}-x_{i j k}\right)\right)=0 \\
\sum_{i=1}^{m} \sum_{j=1}^{n} \sum_{k=1}^{l} z_{i j k} x_{i j k}=0
\end{array} .\right.
$$

Now, the KKT conditions, lead the optimization problem to

$$
\left\{\begin{array}{l}
\operatorname{Max} Z=\sum_{j=1}^{n} S_{j} y_{j}-\sum_{j=1}^{n} \sum_{i=1}^{m} \sum_{k=1}^{l} c_{i j k} x_{i j k}-\sum_{j=1}^{n}\left(h_{j}+p_{j}\right) \sum_{r<y_{j}}\left(y_{j}-r\right) \mu_{j}^{r}+\sum_{j=1}^{n} p_{j} y_{j} \\
\text { subject to } \\
c_{i j k}-u_{i}-v_{k} \leq 0 \\
x_{i j k}\left(c_{i j k}-u_{i}-v_{k}\right)=0 \\
\sum_{j=1}^{n} \sum_{k=1}^{l} x_{i j k} \leq a_{i} \text { for } i=1,2, \ldots, m \\
\sum_{i=1}^{m} \sum_{j=1}^{n} x_{i j k} \leq e_{k} \text { for } k=1,2, \ldots, l \\
x_{i j k} \geq 0 \forall i, j, k
\end{array} .\right.
$$

\section{NUMERICAL EXAMPLE}

In order to show the application of the proposed model, we shall present the phenomenon of coal transportation problem. Coal is a kind of important energy source in the development of economy and society. Accordingly, how to transport coal from mines to different areas is an important issue in coal transportation. For the simplicity of description, we summarize the problem as follows. Suppose that there are three coal mines (i.e., $m=3$ ) to supply the coal in two companies (i.e., $n=2$ ). During the process of transportation, two kinds of conveyances to be selected, i.e., train and cargo ship $(k=2)$. Now, the task for the decision-maker is to make the transportation 
TABLE 2. Input data.

\begin{tabular}{|c|c|c|c|c|c|c|}
\hline Unit transportation cost & & Holding cost & Shortage cost & Availability & Convaynce & Selling price \\
\hline$c_{i j 1}$ & $c_{i j 2}$ & $h_{j}$ & $p_{j}$ & $a_{i}$ & $e_{k}$ & $S_{j}$ \\
\hline$c_{111}=5, c_{211}=5, c_{311}=7$ & $c_{212}=8, c_{112}=11, c_{312}=8$ & $h_{1}=7$ & $p_{1}=18$ & $a_{1}=50, a_{2}=53$ & $e_{1}=100$ & $S_{1}=39$ \\
\hline$c_{121}=6, c_{221}=12, c_{321}=4$ & $c_{122}=9, c_{222}=9, c_{322}=7$ & $h_{2}=9$ & $p_{2}=12$ & $a_{3}=72$ & $e_{2}=75$ & $S_{2}=42$ \\
\hline
\end{tabular}

TABLE 3. Resulting solutions of Sub-Model-1 for different $\alpha_{j}, \beta_{j}$.

\begin{tabular}{|c|c|c|c|c|c|c|c|c|}
\hline Demand parameters & Conveyance $K$ & & & & & Lagrange & Multipliers & $Z$ \\
\hline \multirow{4}{*}{$\begin{array}{l}\alpha_{1}=62, \alpha_{2}=74 \\
\beta_{1}=12, \beta_{2}=18\end{array}$} & $i / j$ & 1 & 2 & 1 & 2 & \multirow{4}{*}{$\begin{array}{l}u_{1}=4.39 \\
u_{2}=8.39\end{array}$} & \multirow{4}{*}{$\begin{array}{l}v_{1}=3.97 \\
v_{2}=7.97\end{array}$} & \multirow{4}{*}{5703.79} \\
\hline & 1 & 0 & 0 & 6 & 38 & & & \\
\hline & 2 & 0 & 49 & 0 & 0 & & & \\
\hline & 3 & 43 & 0 & 25 & 0 & & & \\
\hline \multirow{3}{*}{$\begin{array}{l}\alpha_{1}=78, \alpha_{2}=84 \\
\beta_{1}=16, \beta_{2}=10\end{array}$} & 1 & 0 & 0 & 47 & 0 & \multirow{3}{*}{$\begin{array}{l}u_{1}=4.39 \\
u_{2}=8.39\end{array}$} & \multirow{3}{*}{$\begin{array}{l}v_{1}=3.97 \\
v_{2}=7.97\end{array}$} & \multirow[t]{3}{*}{6157.87} \\
\hline & 2 & 0 & 53 & 0 & 0 & & & \\
\hline & 3 & 40.15 & 6.84 & 0 & 25 & & & \\
\hline \multirow{3}{*}{$\begin{array}{l}\alpha_{1}=79, \alpha_{2}=85 \\
\beta_{1}=17, \beta_{2}=11\end{array}$} & 1 & 0 & 0 & 0 & 47 & \multirow{3}{*}{$\begin{array}{l}u_{1}=4.39 \\
u_{2}=8.39\end{array}$} & \multirow{3}{*}{$\begin{array}{l}v_{1}=3.97 \\
v_{2}=7.97\end{array}$} & \multirow[t]{3}{*}{6477.34} \\
\hline & 2 & 14.65 & 38.34 & 0 & 0 & & & \\
\hline & 3 & 47 & 0 & 25 & 0 & & & \\
\hline
\end{tabular}

TABLE 4. Resulting solutions of Sub-Model-2 for different $\lambda_{j}$.

\begin{tabular}{|c|c|c|c|c|c|c|c|c|}
\hline Demand parameters & Conveyance $K$ & & & & & Lagrange & Multipliers & $Z$ \\
\hline \multirow{4}{*}{$\lambda_{1}=0.018, \lambda_{2}=0.012$} & $i / j$ & 1 & 2 & 1 & 2 & \multirow{4}{*}{$\begin{array}{l}u_{1}=4.67 \\
u_{2}=4.67\end{array}$} & \multirow{4}{*}{$\begin{array}{l}v_{1}=9.32 \\
v_{2}=6.32\end{array}$} & \multirow{4}{*}{4575.41} \\
\hline & 1 & 34.56 & 0 & 0 & 0 & & & \\
\hline & 2 & 0 & 49.34 & 0 & 3.23 & & & \\
\hline & 3 & 0 & 0 & 39 & 31.14 & & & \\
\hline \multirow[t]{3}{*}{$\lambda_{1}=0.015, \lambda_{2}=0.012$} & 1 & 43.19 & 0 & 0 & 6 & \multirow{3}{*}{$\begin{array}{l}u_{1}=4.67 \\
u_{2}=4.67\end{array}$} & \multirow{3}{*}{$\begin{array}{l}v_{1}=9.32 \\
v_{2}=6.32\end{array}$} & \multirow[t]{3}{*}{5209.39} \\
\hline & 2 & 0 & 49.75 & 0 & 0 & & & \\
\hline & 3 & 0 & 0 & 31 & 37 & & & \\
\hline \multirow{3}{*}{$\lambda_{1}=0.014, \lambda_{2}=0.018$} & 1 & 32.25 & 0 & 0 & 0 & \multirow{3}{*}{$\begin{array}{l}u_{1}=4.67 \\
u_{2}=4.67\end{array}$} & \multirow{3}{*}{$\begin{array}{l}v_{1}=9.32 \\
v_{2}=6.32\end{array}$} & \multirow[t]{3}{*}{5914.08} \\
\hline & 2 & 0 & 34 & 18.25 & 0 & & & \\
\hline & 3 & 32.5 & 0 & 0 & 32.8 & & & \\
\hline
\end{tabular}

plan in order to maximize the profit. At the beginning of this problem, the decision maker needs the basic data, such as availability of the origin, demand of the destination, capacity of the conveyances, transportation cost of unit product, and so on. In this regard, the following input values are taken into consideration, common to all the three Sub-Models (Tab. 2 ).

For the above forecast input data, each sub-model, random demand parameters are presented in the first column of the respective Tables $3-5$.

From the above illustration, it is seen that the optimal solutions are basic i.e., the solutions are basic feasible solutions, since the number of non-trivial solutions is $(m+n+l-2)$. From Tables 3 to 5 , it is clear that more expected demand influences the decision-makers to transport more quantity of items. Consequently, more amount transportation increases the total cost of the system as well as profit of the system. Here, it is also seen that most of the Lagrangian multipliers are positive, which indicate for the global optimal solution of the model. 
TABLE 5. Resulting solutions of Sub-Model-3 for different $d_{j}$.

\begin{tabular}{|c|c|c|c|c|c|c|c|c|}
\hline Demand parameters & Conveyance $K$ & & & & & Lagrange & Multipliers & $Z$ \\
\hline & $i / j$ & 1 & 2 & 1 & 2 & \multirow{4}{*}{$\begin{array}{l}u_{1}=4.11 \\
u_{2}=6.11\end{array}$} & \multirow{4}{*}{$\begin{array}{l}v_{1}=2.44 \\
v_{2}=3.10\end{array}$} & \multirow{4}{*}{5600.84} \\
\hline$d_{1}=(57,0.3), d_{2}=(51,0.2)$ & 1 & 37.34 & 0 & 0 & 0 & & & \\
\hline$d_{1}=(58,0.4), d_{2}=(52,0.5)$ & 2 & 0 & 19 & 29.23 & 0 & & & \\
\hline$d_{1}=(59,0.3), d_{2}=(53,0.3)$ & 3 & 0 & 21.56 & 0 & 31.14 & & & \\
\hline$d_{1}=(61,0.3), d_{2}=(54,0.2)$ & 1 & 29.25 & 0 & 20.2 & 0 & \multirow{3}{*}{$\begin{array}{l}u_{1}=4.11 \\
u_{2}=6.11\end{array}$} & \multirow{3}{*}{$\begin{array}{l}v_{1}=2.44 \\
v_{2}=3.10\end{array}$} & \multirow[t]{3}{*}{6189.45} \\
\hline$d_{1}=(62,0.4), d_{2}=(55,0.5)$ & 2 & 0 & 46 & 0 & 0 & & & \\
\hline$d_{1}=(63,0.3), d_{2}=(56,0.3)$ & 3 & 23.5 & 0 & 0 & 31.6 & & & \\
\hline$d_{1}=(59,0.3), d_{2}=(59,0.2)$ & 1 & 0 & 49.75 & 0 & 0 & \multirow{3}{*}{$\begin{array}{l}u_{1}=4.11 \\
u_{2}=6.11\end{array}$} & $v_{1}=2.44$ & \multirow[t]{3}{*}{6554.3} \\
\hline$d_{1}=(60,0.4), d_{2}=(60,0.5)$ & 2 & 38.1 & 0 & 12 & 0 & & $v_{2}=3.10$ & \\
\hline$d_{1}=(61,0.3), d_{2}=(61,0.3)$ & 3 & 0 & 0 & 24.2 & 37 & & & \\
\hline
\end{tabular}

\section{Practical implementation}

In developing countries, like India, Bangladesh, Nepal, China, etc., due to several reasons, the parameters especially the demand of an item is uncertain in nature. For these reasons, if we collect the previous data from any management system belong to these countries and followed by statistical regularity criteria, its probability distribution can be obtained for future correspondence. For this type of random demand with exponential and logistic distribution, has a wide range of applications ( $c f .[22]$ ) has been considered here. Not only that, the more realistic discrete demand distribution is taken here. Such type of transportation system is found for the seasons products, like-winter garments, raincoats, summer creams, seasonal fruit juice, etc., or the attractive items, like bikes, cars, and mobile phones, etc. Here as per reality, profit maximization criteria are introduced instead of cost minimization of the system. So the above transportation model has a wide practical area of the above mentioned transportation management.

\section{Discussion AND CONCLUSION}

In today's highly comparative market, the decision-makers deliver the product to the customer in effective ways, although the system has a heavy uncertainty. The literature focused on a solid transportation problem (STP) where the demand of the destinations are not fixed quantities, but uncertain in nature. The presented numerical result is a choice of such decision making problem for a particular illustration. Here such a STP is taken into consideration with a more realistic profit maximization form. The formulated STP is simplified with different probability distributions and their comparative results are illustrated.

The proposed model is one of the realistic models, so it can be developed with other different types of environments, like rough, fuzzy rough, fuzzy stochastic, etc. The model can be extended with a multi-item solid transportation problem, model with fixed charge, model with more vehicle costs, etc. The proposed SSTP can be solved with some other programming problems, like genetic algorithms, simulation methods, etc. All these can be taken as future research.

Acknowledgements. The authors heartily acknowledge the financial grant provided to the first author as INSPIRE fellow [No. IF150344] by the Department of Science and Technology, Government of India. The authors also would like to show their gratitude to the anonymous referees for their constructive and motivating comments and suggestions for the improvement of the article. Further, the authors express their warm gratitude to the Editor and Associate Editor for considering this article for publication.

\section{REFERENCES}

[1] H.G. Akdemir and F. Tiryaki, Bilevel stochastic transportation problem with exponentially distributed demand. Bitlis Eren Univ. J. Sci. Technol. 2 (2012) 32-37. 
[2] P. Anukokila, B. Radhakrishnan and A. Anju, Goal programming approach for solving multi-objective fractional transportation problem with fuzzy parameters. RAIRO: OR $\mathbf{5 3}$ (2019) 157-178.

[3] A.K. Bit, M.P. Biswal and S.S. Alam, Fuzzy programming approach to multi-objective solid transportation problem. Fuzzy Sets Syst. 57 (1993) 183-194.

[4] A.K. Bhurjee and G. Panda, Multi-objective optimization problem with bounded parameters. RAIRO: OR 48 (2014) 545-558.

[5] L. Cooper and L.J. LeBlanc, Stochastic transportation problems and other network related convex problems. Nav. Res. Logistics Q. 24 (1977) 327-336.

[6] A. Das, U.K. Bera and B. Das, A solid transportation problem with mixed constraint in different environment. J. Appl. Anal. Comput. 6 (2016) 179-195.

[7] S.K. Das, T. Mandal and S.A. Edalatpanah, A new approach for solving fully fuzzy linear fractional programming problems using the multi-objective linear programming. RAIRO: OR 51 (2017) 285-297.

[8] U. Habiba and A. Quddoos, Multiobjective stochastic interval transportation problem involving general form of distributions. Adv. Math. Sci. J. 9 (2020) 3213-3219.

[9] S. Halder, B. Das, G. Panigrahi and M. Maiti, Solving a solid transportation problems through fuzzy ranking. In: Communication, Devices, and Computing. Springer, Singapore (2017) 283-292.

[10] K. Haley, The solid transportation problem. Oper. Res. 10 (1962) 448-463.

[11] K. Holmberg, Separable programming applied to the stochastic transportation problem. Research Report, LITH-MAT-R-198415. Department of Mathematics, Linkoping Institute of Technology, Sweden (1984).

[12] K. Holemberg and K.O. Jornsten, Cross decomposition applied to the stochastic transportation problem. Eur. J. Oper. Res. 17 (1984) 361-368.

[13] K. Holemberg and H. Tuy, A production-transportation problem with stochastic demand and concave production costs. Math. Program. 85 (1999) 157-179.

[14] S. Jana, B. Das, G. Panigrahi and M. Maiti, Profit Maximization solid transportation problem with Gaussian type-2 fuzzy environments. Ann. Pure Appl. Math. 2 (2018) 323-335.

[15] G.R. Jahanshahloo, B. Talebian, I.H. Lotfi and J. Sadeghi, Finding a solution for multi-objective linear fractional programming problem based on goal programming and data envelopment analysis. RAIRO: OR 51 (2017) 199-210.

[16] F. Jimnez and J.L. Verdegay, Uncertain solid transportation problems. Fuzzy Sets Syst. 100 (1998) 45-57.

[17] D.S. Kim and N.V. Tuyen, A note on second-order Karush-Kuhn-Tucker necessary optimality conditions for smooth vector optimization problems. RAIRO:OR 52 (2018) 567-575.

[18] A. Kuiri and B. Das, A non-linear transportation problem with additional constraints in fuzzy environment. J. Emerg. Technol. Innov. Res. 5 (2018) 277-283.

[19] A. Kuiri and B. Das, An application of dynamic programming problem in multi stage transportation problem with fuzzy random parameters. IOSR J. Eng. 9 (2019) 30-41.

[20] D.R. Mahapatra, Multi-choice stochastic transportation problem involving Weibull distribution. Int. J. Optim. Control Theor. App. 4 (2014) 45-55.

[21] D. Mahapatra, S. Roy and M. Biswal, Computation of multi-objective stochastic transportation problem involving normal distribution with joint constraints. J. Fuzzy Math. 19 (2011) 865-876.

[22] D.R. Mahapatra, S.K. Roy and M.P. Biswal, Multi-choice stochastic transportation problem involving extreme value distribution. Appl. Math. Modell. 37 (2013) 2230-2240.

[23] G. Maity and S.K. Roy, Multi-item multi-choice integrated optimisation in inventory transportation problem with stochastic supply. Int. J. Oper. Res. 35 (2019) 318-339.

[24] G. Maity, S.K. Roy and J.L. Verdegay, Analyzing multimodal transportation problem and its application to artificial intelligence. Neural Comput. App. 32 (2020) 2243-2256.

[25] S. Midya and S.K. Roy, Solving single-sink, fixed-charge, multi-objective, multi-index stochastic transportation problem. Am. J. Math. Manage. Sci. 33 (2014) 300-314.

[26] A. Nagarjan and K. Jeyaraman, Solution of chance constrained programming problem for multi-objective interval solid transportation problem under stochastic environment using fuzzy approach. Int. J. Comput. App. 10 (2010) 19-29.

[27] A.K. Ojha and R. Ranjan, Multi-objective geometric programming problem with Karush-Kuhn-Tucker condition using $\epsilon$ constraint method. RAIRO: OR 48 (2014) 429-453.

[28] A. Ojha, B. Das, S. Mondal and M. Maiti, A stochastic discounted multi-objective solid transportation problem for breakable items using analytical hierarchy process. Appl. Math. Modell. 34 (2010) 2256-2271.

[29] A. Ojha, B. Das, S. Mondal and M. Maiti, A solid transportation problem for an item with fixed charge, vechicle cost and price discounted varying charge using genetic algorithm. Appl. Soft Comput. 10 (2010) 100-110.

[30] A. Quddoos, G. Hasan and M.M. Khalid, Multi-choice stochastic transportation problem involving general form of distributions. SpringerPlus 3 (2014) 1-9.

[31] S.K. Roy, Transportation problem with multi-choice cost and demand and stochastic supply. J. Oper. Res. Soc. Ch. 4 (2016) 193-204.

[32] S.K. Roy and S. Midya, Multi-objective fixed-charge solid transportation problem with product blending under intuitionistic fuzzy environment. Appl. Intell. 49 (2019) 3524-3538.

[33] S.K. Roy, D.R. Mahapatra and M.P. Biswal, Multi-choice stochastic transportation problem with exponential distribution. J. Uncertain Syst. 6 (2012) 200-213. 
[34] S.K. Roy, S. Midya and V.F. Yu, Multi-objective fixed-charge transportation problem with random rough variables. Int. J. Uncertainty Fuzziness Knowledge-Based Syst. 26 (2018) 971-996.

[35] S.K. Roy, S. Midya and G.W. Weber, Multi-objective multi-item fixed-charge solid transportation problem under twofold uncertainty. Neural Comput. App. 31 (2019) 8593-8613.

[36] S. Samanta, B. Das and S.K. Mondal, A new method for solving a fuzzy solid transportation model with fuzzy ranking. Asian J. Math. Phys. 2 (2018) 73-83.

[37] A.C. Williams, A stochastic transportation problem. Oper. Res. 11 (1963) 759-770.

[38] D. Wilson, An a priori bounded model for transportation problems with stochastic demand and integer solutions. AIIE Trans. 4 (1972) 186-193.

[39] L. Yang and Y. Feng, A bicriteria solid transportation problem with fixed charge under stochastic environment. Appl. Math. Modell. 31 (2007) 2668-2683.

[40] L. Yang, P. Liu, S. Li, Y. Gao and D.A. Ralescu, Reduction methods of type-2 uncertain variables and their applications to solid transportation problem. Inf. Sci. 291 (2015) 204-237. 